\title{
Optimization of Stability Period in WSN using GA based Stable Election Protocol
}

\author{
Mandeep Kaur \\ M.Tech Scholar \\ GNDEC, Ldh
}

\author{
Kanwaljit Singh \\ Prof. ECE Deptt \\ GNDEC, Ldh
}

\begin{abstract}
Wireless sensor network is an emerging and new technology in the field of data processing and wireless communication. The wireless network is composed of tiny nodes called as sensor nodes which are battery powered and are capable of transferring information to the destination i.e. Sink. Being battery powered these nodes are limited in energy and loose the energy during the process of routing. Once the sensor node has lost all its energy, it is dead. With the death of first sensor node the network becomes unstable. The stable election protocol (SEP) aims to increase the stability period of the network i.e. the period before the death of the first node. In this paper we propose the optimization of the stability period the sensor network and optimizing tool used here is genetic algorithm (GA). For the proficiency in stability period, the GA is applied on SEP. The simulation of the proposed algorithm is done on MATLAB and the results are compared with heterogeneous LEACH and SEP.
\end{abstract}

\section{Keywords}

Clustering, GA, Optimization, SEP, Stability period, WSNs.

\section{INTRODUCTION}

Wireless sensor network is a widely developing field and find applications in different areas such as military-target tracing, habitat monitoring, environmental conditions monitoring, security management etc. A wireless sensor network is a collection of tiny sensor nodes that are capable of monitoring and processing the surrounding geographical area. These Sensor nodes are deployed very densely inside the phenomenon or very close to it, where the position of these nodes needs not to be pre-defined or engineered [1], [2]. This allows the random deployment of the sensor network in the area of interest. The number of nodes deployed in WSN can vary from tens to tens of thousands depending on the particular application. Their positions can be static or mobile.

The tiny sensor nodes are battery powered and are equipped with their own sensor processor and radio transceiver for communicating wirelessly [13]. In WSNs to utilize the energy of the network efficiently number of protocols has been developed that are based on direct transmission (DT) or minimum transmission energy (MTE) or clustering based protocols. In case of direct transmission (DT) the nodes transmit data directly to the sink, as a result the nodes that are far away from the sink die fast [12]. On the other hand, in minimum transmission energy, data is relayed onto the minimum cost route, i.e. the path on which least power is consumed. As the nodes near to the sink act as relay, tend to die fast. Thus the design of the protocol for the WSNs should be such that it should be energy aware and helpful in maximizing the network lifetime. A solution to this problem is LEACH (Low-energy adaptive clustering hierarchy) protocol that guarantees well distribution of energy amongst all the nodes by dynamically created clusters \& cluster heads dynamically elected according to optimal probability.

LEACH is a hierarchical clustering algorithm that performs well in homogeneous WSN. A homogeneous WSN is one in which all nodes are identical in terms of energy and have equal probability to become cluster head. Amongst all the nodes the cluster heads are elected randomly and the role of each node becoming cluster head is rotated periodically according to a random number between 0 and 1 [14]. A node can become cluster head for the current rotation if the number is less than the following threshold:

$\mathrm{T}(\mathrm{s})=\left\{\begin{array}{cc}\frac{p}{1-p *\left(r * \bmod \left(\frac{1}{p}\right)\right.} \quad \mathrm{s} \in \mathrm{G} \\ 0 \quad \text { otherwise }\end{array}\right.$

where $\mathrm{p}$ is the desired percentage of the $\mathrm{CH}$ nodes in the sensor population, $r$ is the current round number, and $\mathrm{G}$ is the set of nodes that have not been $\mathrm{CHs}$ in the last $1 / \mathrm{p}$ rounds. However, LEACH is not well suited for heterogeneous environment [4]. SEP is two level heterogeneous protocol that introduces two types of nodes i.e. normal nodes and advanced nodes. A fraction $m$ (advanced nodes) of total nodes $n$ is provided with an additional energy factor $\alpha$. So due to the presence of advanced nodes the stability period of the sensor network is increased.

\section{Paper Organization}

Rest of the paper is organized as: section 2 describes the standard stable election protocol. In section 3 we describe our GA based optimized protocol. Section 4 explains the communication model for the wireless sensor network. In section 5 simulation results of our proposed protocol are given compared with other protocols. Finally the paper is concluded in section 6

\section{STABLE ELECTION PROTOCOL (SEP)}

In SEP, the probability of each node becoming $\mathrm{CH}$ is weighted by initial energy of node relative to that of other nodes in the network. Suppose $\mathrm{E}_{0}$ is the initial energy of each normal node. The energy of each advanced node is then $\mathrm{E}_{0}(1+\alpha)$. Also:

$$
\begin{gathered}
p_{\text {nrm }}=p /(1+\alpha * m) \\
p_{a d v}=(p /(1+\alpha * m)) *(1+\alpha)
\end{gathered}
$$

and the threshold in SEP is replaced by threshold for normal sensor, $\mathrm{T}\left(s_{n r m}\right)$, and threshold for advanced sensor, $\mathrm{T}\left(s_{a d v}\right)$, as follows:

$$
\mathrm{T}\left(s_{\text {nrm }}\right)=\left\{\begin{array}{c}
\frac{p_{\text {nrm }}}{1-p_{\text {nrm }} *\left(r * \bmod \left(1 / p_{\text {nrm }}\right)\right.} \\
0 \quad \text { if } s \in G^{\prime} \\
0 \text { otherwise }
\end{array}\right.
$$




$$
\mathrm{T}\left(s_{a d v}\right)=\left\{\begin{array}{cl}
\frac{p_{a d v}}{1-p_{a d v} *\left(r * \bmod \left(1 / p_{a d v}\right)\right.} & \text { if } s \in G^{\prime \prime} \\
0 & \text { otherwise }
\end{array}\right.
$$

where $r$ is the current round, $G^{\prime}$ is the set of normal nodes that have not become cluster heads within the last $1 / p_{n r m}$ rounds of the epoch, and $\mathrm{T}\left(\left(s_{n r m}\right)\right.$ is the threshold applied to a population of $\mathrm{n} *(1-\mathrm{m})$ normal nodes. This guarantees that each normal node will become a cluster head exactly once every $1 / \mathrm{p}^{*}\left(1+\alpha^{*} \mathrm{~m}\right)$ rounds and that the average number of cluster heads that are normal nodes per round is equal to $\mathrm{n} *(1-\mathrm{m}) * p_{n r m}$. Similarly, G' ' is the set of advanced nodes that have not become cluster heads within the last $1 / p_{a d v}$ rounds of the epoch, and $\mathrm{T}\left(s_{a d v}\right)$ is the threshold applied to a population of $n * m$ advanced nodes. This guarantees that each advanced node will become a cluster head exactly once every $\left.\left.\left((1) /(\mathrm{p}) \times\left(\left(1+\alpha^{*} \mathrm{~m}\right)\right) /(1+\alpha)\right)\right)\right)$ rounds [8]. Being a heterogeneity protocol SEP aims to improve the stability period and the network lifetime of the WSN. But it has a demerit that the throughput is also increased causing decrease in network lifetime [12]. Our proposed algorithm is observed to be better option to control the tradeoff between energy efficiency, accuracy and response time.

\section{GENETIC ALGORITHM}

Genetic Algorithm is used to create cluster members, cluster heads and next clusters dynamically, which evaluate the average fitness of the system and is used to increase the network lifetime. In [5], Hussain and Matin described the genetic algorithm for dynamic clustering in WSNs. In the WSNs each node is described by a chromosome, the value of which is specified by binary bits ' 0 ' or ' 1 '. If the value is ' 1 ' the corresponding node is an advanced node else it is a regular node. For the optimization of the stability the genetic algorithm is applied on SEP. The main features of genetic algorithm are: crossover, mutation and fitness function.

\subsection{Population}

A population is collection of several chromosomes and the best chromosome is used to generate the next population. Initially the GA starts with a population of predefined number of chromosomes and randomly selected cluster heads. Then each chromosome is evaluated by GA by calculating its fitness. After evaluating the fitness GA selects best suitable chromosome and then applies crossover and mutation operators.

\subsection{Fitness}

The fitness of a chromosome is its ability to pass on its genetic material \& also its quality to survive. The fitness of the chromosome is designed to minimize the energy consumption and to increase the network lifetime. The fitness function of the chromosome is defined as:

$\mathrm{F}=\left(w_{i}, f_{i}\right) \forall f_{i} \in\{C, D, E, S D, T\}$

The initial weight $\mathrm{s}$ of the fitness function are selected arbitrarily, which are updated in each round to select the best chromosome. In the fitness function the term $\mathrm{D}$ is defined as direct distance and is the sum of all the distances from cluster nodes to the sink. C (Cluster Distance) is the sum of distances from nodes to $\mathrm{CH}$ and from $\mathrm{CH}$ to the sink. $\mathrm{D}=\sum_{i=1}^{m} \boldsymbol{d}_{\boldsymbol{i}}$.

$\mathrm{C}$ (Cluster Distance) is the sum of distances from nodes to $\mathrm{CH}$ and from $\mathrm{CH}$ to the sink. $\mathrm{C}=\sum_{\boldsymbol{i}=\mathbf{1}}^{\boldsymbol{k}} \boldsymbol{d}_{\boldsymbol{i}}+\boldsymbol{d}_{\boldsymbol{h}}$ where $\boldsymbol{d}_{\boldsymbol{i}}$ is the distance from the node $i$ to the cluster head and $d_{h}$ is the distance from the cluster head to the sink.

E (Transfer Energy) is the energy consumed by the network to transfer the aggregated data from cluster to the base station where a part of the total transfer energy is consumed to transfer the message from node to cluster head, another part is consumed by the cluster head to receive k-bit message and finally by the cluster head to transfer the message to the base station (sink). SD is the cluster distance Standard Deviation and $\mathrm{T}$ defines the number of transmissions assigned by the base station for each data transfer stage. Initially the fitness parameters are assigned arbitrary weights and after every aggregation these weights are updated and best fit chromosome is evaluated to produce the next generation. In [8] \& [9], Attea and Khalil described the Genetic algorithm for WSNs with improved fitness function by redefining the distance function.

\subsection{Selection}

Selection is the process of determining which two chromosomes will mate to form a new chromosome. The chromosomes with higher fitness values have more chances to of matting.

\subsection{Crossover}

Crossover is a binary genetic operation applied on two chromosomes. Crossover recombines the genetic materials of two parent chromosomes to produce a child chromosome. The results of the crossover depend on the selection procedure.

\subsection{Mutation}

In mutation the genetic material of individual changes of its own. The mutation adds variations in the next generation.

\section{WIRELESS COMMUNICATION MODEL}

According to the communication model [4] of wireless sensor networks the energy consumed to transmit a k-bit message over a distance $d$ is given by:

$E_{T x}(\mathrm{k}, \mathrm{d})= \begin{cases}k * E_{\text {elec }}+k * E_{f c} d^{2} & d \leq d_{0} \\ k * E_{\text {elec }}+k * E_{m p} d^{4} & d>d_{0}\end{cases}$

Where $\mathrm{d}_{0}$ is the short distance defined as $d_{0}=\sqrt{E_{f c} / E_{m p}}$ and $E_{\text {elec }}=50 \mathrm{nl} / \mathrm{bit}$. Then the energy consumed to receive the above k-bit message is given by $E_{R x}(\mathrm{k}, \mathrm{d})=\mathrm{k}^{*} E_{\text {elec }}$

\section{SIMULATION RESULTS}

In this section we investigate our GA based SEP protocol against LEACH and SEP. The results of simulation show that our proposed protocol is better than other protocols in terms of stability period of the network

\subsection{Simulation Settings}

The different routing protocols are implemented in MATLAB. The simulation is performed on different sensor networks, each composed of 100 sensor nodes deployed randomly in an area of $100 \mathrm{~m} \times 100 \mathrm{~m}$, assuming sink to be located at the center of the sensor network. The initial energy of a normal node is set as $E_{0}=0.5 \mathrm{~J}, E_{f c}=10 \mathrm{pJ} / \mathrm{bit} / \mathrm{m}^{2}, E_{m p}=$ $0.0013 \mathrm{pJ} / \mathrm{bit} / \mathrm{m}^{4}$ and $E_{D A}=5 \mathrm{~nJ} / \mathrm{bit} /$ report. The message length is taken to be 4000 bits.

Table1 shows the comparison of different protocols with $10 \%$ advanced nodes and $\alpha=2$ and Table 2 shows the comparison with $20 \%$ advanced nodes and $\alpha=1$. 
Table1. Comparison of LEACH, SEP and GASEP with $\mathrm{m}=0.1$ and $\alpha=2$

\begin{tabular}{|c|c|c|c|c|c|c|}
\hline S. No. & \multicolumn{2}{|c|}{ LEACH } & \multicolumn{2}{c|}{ SEP } & \multicolumn{2}{c|}{ GASEP } \\
\hline & FND & LND & FND & LND & FND & LND \\
\hline 1 & 1025 & 4353 & 1122 & 3032 & 2282 & 3455 \\
\hline 2 & 1030 & 5148 & 1069 & 3707 & 2280 & 3359 \\
\hline 3 & 1043 & 3724 & 1131 & 3486 & 2218 & 3396 \\
\hline 4 & 1048 & 4388 & 1133 & 3715 & 2322 & 3501 \\
\hline 5 & 1068 & 5502 & 1098 & 3212 & 2208 & 3456 \\
\hline
\end{tabular}

Table 2. Comparison of LEACH, SEP and GASEP with $\mathrm{m}=0.2$ and $\alpha=1$

\begin{tabular}{|c|c|c|c|c|c|c|}
\hline S. No. & \multicolumn{2}{|c|}{ LEACH } & \multicolumn{2}{c|}{ SEP } & \multicolumn{2}{c|}{ GASEP } \\
\hline & FND & LND & FND & LND & FND & LND \\
\hline 1 & 996 & 4851 & 1173 & 3130 & 2250 & 3378 \\
\hline 2 & 942 & 6759 & 1078 & 3862 & 2258 & 3367 \\
\hline 3 & 1003 & 6414 & 1145 & 3752 & 2260 & 3452 \\
\hline 4 & 954 & 6138 & 1063 & 4218 & 2218 & 3383 \\
\hline 5 & 981 & 6792 & 1176 & 3239 & 2208 & 3414 \\
\hline
\end{tabular}

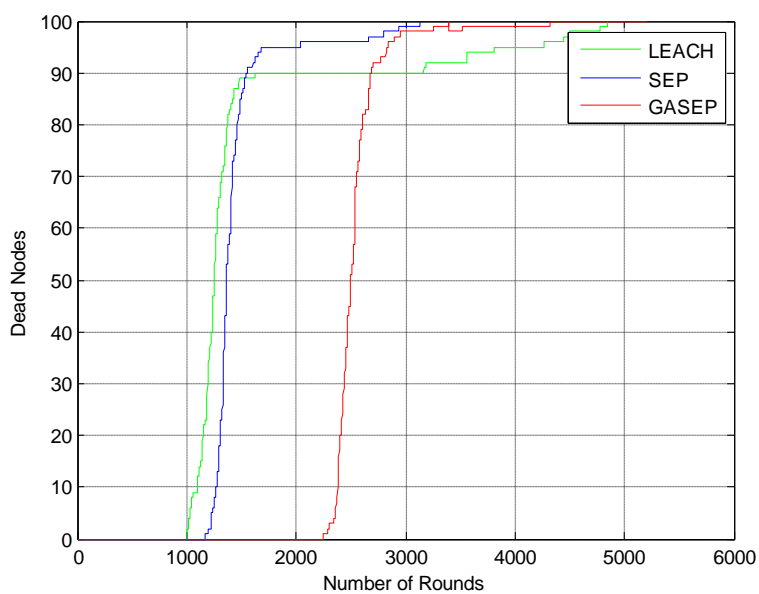

Fig 1: Stability of LEACH, SEP and GASEP with $m=0.1$ and $\alpha=2$

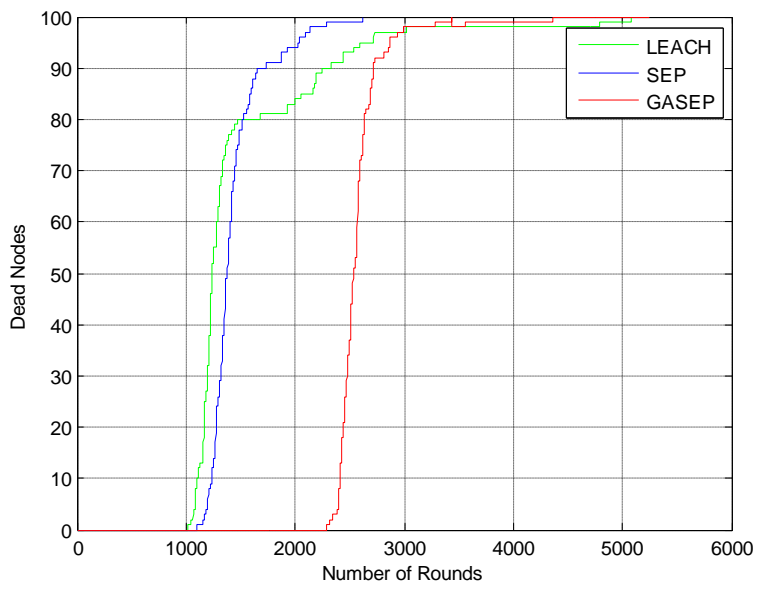

Fig 2. Stability of LEACH, SEP and GASEP with $\mathrm{m}=0.2$ and $\alpha=1$

\section{CONCLUSION}

This paper concludes that the results of our protocol are better than that of heterogeneous LEACH and SEP. In this paper we tried to improve the network stability using genetic algorithm. Furthermore, in this proposed protocol we used two levels of heterogeneity and only two types of nodes (i.e. normal nodes and advanced nodes) are used.

In future the protocol can be further modified to improve the network lifetime too. Moreover the results of the protocol can be further modified by considering three levels of heterogeneity.

\section{REFERENCES}

[1] Akkaya, K., \& Younis, M. (2005). "A survey on routing protocol for wireless sensor networks". Ad hoc Networks 3 , 325-349.

[2] Akyildiz, I., W.Su, Y.Sankarasubramaniam, \& E.Cayirci. (2002). "Wireless Sensor Networks: a Survey". Computer Networks 38 , 393-422.

[3] Faisal, S.,et al (2013). "Z-SEP: Zonal-Stable Election Protocol for Wireless Sensor Networks." arXiv preprint arXiv:1303.5364.

[4] Heinzelman, W. R., Chandrakasan, A., \& Balakrishnan, H. (2000). "Energy-Efficient Communication Protocol for Wireless Microsensor Networks". Hawaii International Conference on System Sciences. Maui, Hwaii: IEEE.

[5] Hussain, S., Matin, A. W., \& Islam, O. (2007). "Genetic Algorithm for Energy Efficient Cluster in Wireless Sensor Networks". Journal of Networks 2 , 87-97.

[6] Jin, Shiyuan, Ming Zhou, \& Annie S. Wu. (2003) "Sensor network optimization using a genetic algorithm." Proceedings of the 7th World Multiconference on Systemics, Cybernetics and Informatics.

[7] Kashaf A, J. Z. (2012). "TSEP: Threshold-sensitive Stable Election Protocol for WSNs". 10th IEEE international conference on frontiers of information.

[8] Khalil, E. A., \& Attea, B. A. (2012). "A new evolutionary based routing protocol for clustered heterogeneous wireless sensor networks". Applied Computing 12, 1950-1957.

[9] Khalil, E. A., \& Attea, B. A. (2011). "Energy-aware evolutionary routing protocol for dynamic clustering of wireless sensor networks". Swarm and Evolutionary Computation 1, 195-203.

[10] Liu, J. L., \& Ravishankar, C. V. (2011). "LEACHGA: Genetic Algorithm based Energy Efficient Adaptive Clustering Protocol for Wireless Sensor Networks". Intrnational Journal of Machine Learning and Computing, Vol. 1, No. 1.

[11] Norouzi, Ali, Faezeh Sadat Babamir, \& Abdul Halim Zaim (2011). "A New Clustering Protocol for Wireless Sensor Networks Using Genetic Algorithm Approach."Wireless Sensor Network 3. 
[12] Samagdakis G., M. I. (2004). "SEP: A Stable Election Protocol for Clustered heterogeneous wireless sensor networks". Boston University Computer Science Department. Toor, A. S., \& kaur,

[13] T. (2013). "Implementation and Analysis of Stable Election Protocol". International Journal of
Advanced Research in Computer Science and Software Engineering , 3 (9), 927-931.

[14] Tyagi, S., \& Kumar, N. (2013). "A systematic review on clustering and routing techniques based upon LEACH protocol for wireless sensor networks". Journal of Network and Computer Applications , 623-645. 